\title{
The Baldwin-effect in Wolf-Rayet stars
}

\author{
Jeroen I. van Gent \\ Astronomy Department, Utrecht University, \\ PO Box $80000, N L-3508$ TA Utrecht, the Netherlands
}

\begin{abstract}
Wolf-Rayet model stellar atmospheres are used to show the relation between He II line equivalent widths and continuum luminosity. It is shown that the Baldwin-effect is present in the models. A comparison made to observational data and model calculations by others stimulates further research into the physics of the effect.
\end{abstract}

\section{Introduction}

An inverse proportionality relation between spectral line equivalent width and continuum luminosity was first found by Baldwin (1977) for quasars. The effect later appeared not to be uniquely confined to quasars, but was shown also to exist in Wolf-Rayet stars. The effect was also brought to light in helium model atmospheres (Morris et al. 1993).

For optically thin lines the Baldwin-effect is explained as resulting from differences in wind density among WR stars. The radius at which the continuum radiation is formed moves further out into the wind when the wind density is increased. Because of this the line emission increases less than the continuum emission, showing up as a decreasing line equivalent width with increasing continuum luminosity (Morris et al. 1993).

In this paper the existence of the Baldwin-effect in WR star model atmospheres is confirmed. In section 2 the model atmospheres are described and the results are discussed. Some remarks on future work conclude this paper.

\section{Model calculations and results}

For the model stellar atmosphere calculations the ISA-Wind code of de Koter (1993) was used. Two grids of models were calculated for a range of atmosphere core radii, core temperatures and for two different mass loss rates. One grid represents more or less early type WN stars (WNE), the second contains late type WN models (WNL).

The chemical elements taken into account in the atmosphere calculations are $\mathrm{H}, \mathrm{He}, \mathrm{C}, \mathrm{N}, \mathrm{O}$, and $\mathrm{Si}$. All elements are treated in non-LTE. The main input parameters of the calculated model grid are presented in Table 1.

Figure 1 shows the relation between equivalent width and the luminosity of the underlying continuum for both WNE and WNL type models for He II transitions. Clearly the Baldwin-effect is present. 
Table 1. Main model input parameters

$$
R_{\text {core }}\left(\mathrm{R}_{\odot}\right) \quad T_{\text {core }}(\mathrm{kK}) \quad \dot{M}\left(10^{-5} \mathrm{M}_{\odot} \mathrm{yr}^{-1}\right) \quad v_{\infty}\left(\mathrm{km} \mathrm{s}^{-1}\right)
$$

$\begin{array}{lcccc}\text { models of figure } 1 \mathrm{a} & 1 \ldots 10 & 35 \ldots 100 & 3,6 & 2500 \\ \text { models of figure 1b } & 12 \ldots 25 & 35 . .47 & 3,6 & 2500 \\ \text { models of figure } 2 & 1 \ldots 30 & 30 \ldots 90 & 5 & 2500\end{array}$
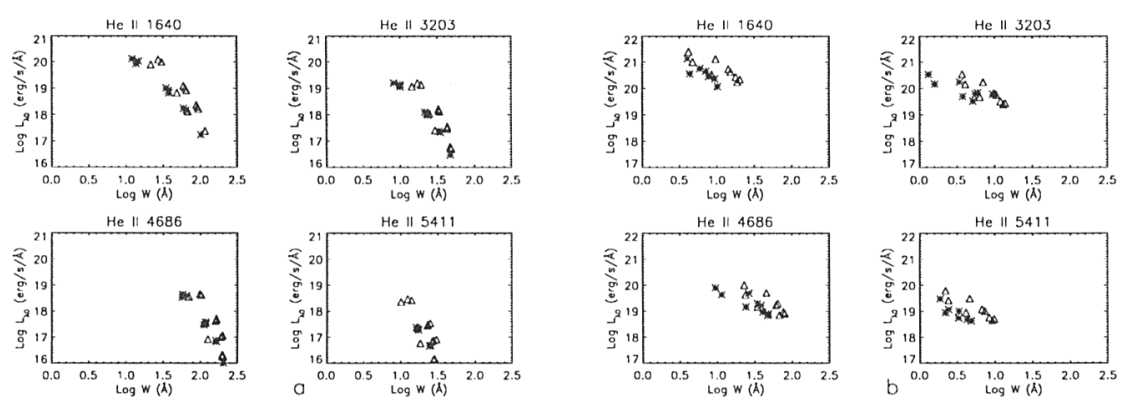

Figure 1. Log continuum luminosity $L$ versus $\log E W$ for He II spectral lines. (a) WNE stars; (b) WNL stars. Stars represent models with mass loss rate $\dot{M}=3 \times 10^{-5} \mathrm{M}_{\odot} \mathrm{yr}^{-1}$; triangles are models with $\dot{M}=6 \times 10^{-5} \mathrm{M}_{\odot} \mathrm{yr}^{-1}$.

For He II transitions, Morris et al. (1993) showed that the Baldwin effect is also present in the non-LTE pure helium atmosphere models from Hamann \& Schmutz (1987) and Schmutz, Hamann \& Wessolowski (1989). This is shown in Figure 2, together with the Baldwin-effect as observed for the LMC WN stars.

As for the helium models, no clear influence of temperature was observed in the ISA-Wind models. The ISA-Wind models with the weakest continua and strongest lines have the smallest radii, confirming the result from Morris et al. (1993) that the Baldwin-effect is largely caused by different wind densities among stars.

The observations show a larger spread than the helium models. The ISAwind models show that models with a larger mass loss rate tend to have a larger line equivalent width at a certain continuum luminosity. Therefore different mass loss rates may be the main cause for the spread in the observational data.

The slope of the Baldwin relation in the ISA-Wind WNL models agrees quite well with that in the helium models. For the WNE model stars the slope is about two times as steep. The physics of this is the subject of current research.

\section{Concluding remarks}

The Baldwin-effect is generally present in WR model atmospheres. Therefore further research is currently done into the details of this effect. 


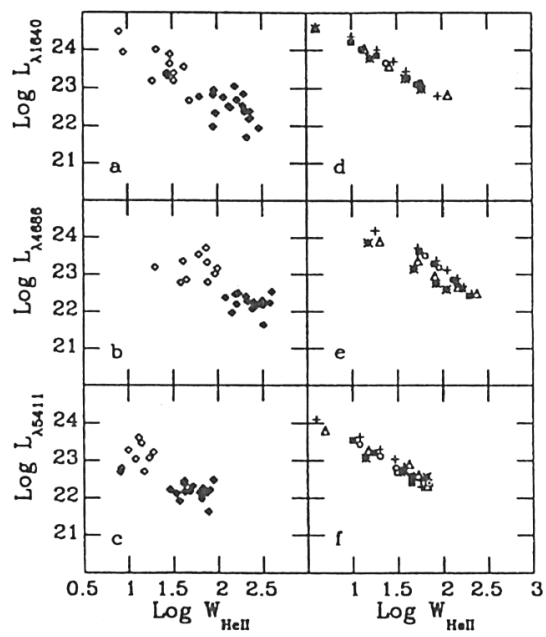

Figure 2. The Baldwin-effect from observed spectra of the LMC WN stars $(a-c)$, together with calculations from pure helium non-LTE model atmospheres $(d-f)$. Open and filled diamonds are WNE and WNL stars, respectively. The other symbols denote effective temperatures: $35 \mathrm{kK}$ (open circles), $45 \mathrm{kK}$ (filled boxes), $55 \mathrm{kK}$ (crosses), $75 \mathrm{kK}$ (triangles), and $90 \mathrm{kK}$ (stars). Figure from Morris et al. (1993).

\section{References}

Baldwin, J.A. 1977, ApJ 214, 679

Hamann, W.-R., Schmutz, W. 1987 A\&A 174, 173

de Koter, A., Schmutz, W., Lamers, H. 1993, A\&A 277, 561

Morris, P.W., Conti, P.S., Lamers, H., Koenigsberger, G. 1993, ApJ 414, L25

Schmutz, W., Hamann, W.-R., Wessolowski, U. 1989, A\&A 210, 236

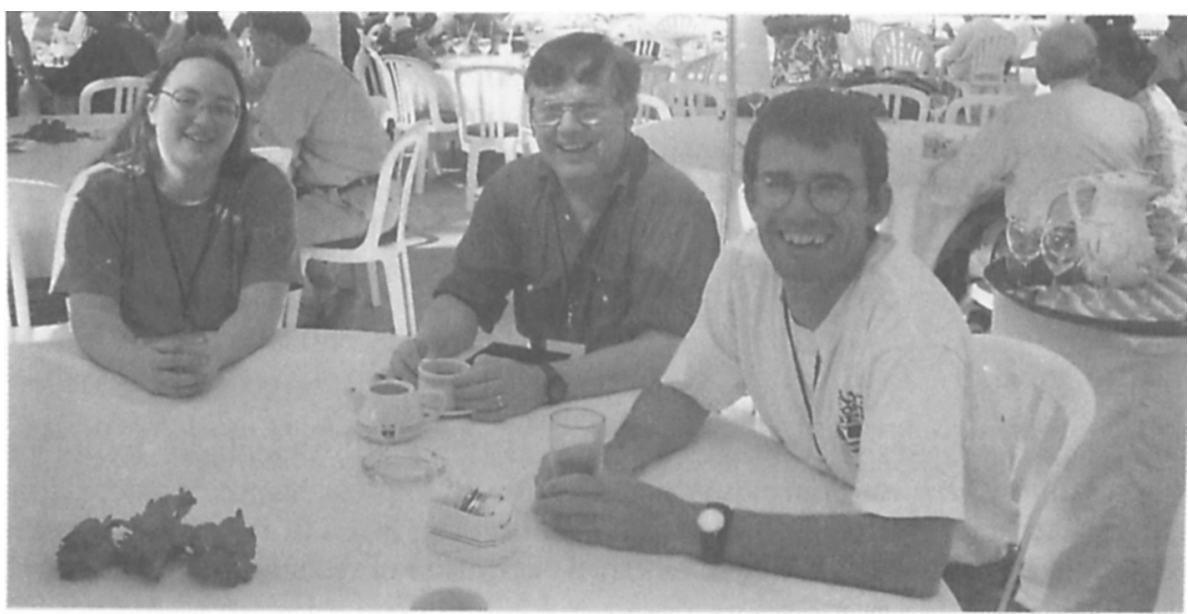

